\title{
Health Literacy and Public Health: what now?
}

\author{
Marco Del Riccio ${ }^{1}$, Gug lielmo Bonaccorsi ${ }^{1}$, Francesca Gigola ${ }^{2}$ \\ 1 University of Florence \\ 2 University of Brescia
}

\begin{abstract}
Even if health literacy has been considered, in the beginning, as an individual-level construct, public health literacy has recently gained more and more importance, and it takes into account the complex social, ecologic, and systemic forces affecting health and well-being.

Is it so possible to measure HL in its different dimensions? How can we measure it at an individual, community, organizational level? The HL measurement tools can explore many different settings, and in the future they will be a key instrument for healthcare organizations to shift to a real citizen-centered vision.
\end{abstract}

\section{Definitions}

\author{
Health Literate Health Care Organizations \\ Defined by Institute of Medicine et al. \\ Public Health Literacy \\ Defined by Darcy A Freedman
}

In a previous article published on the platform, we introduced the concept of individual and Public Health Literacy $(\mathrm{HL})$, highlighting the fact that it is not just a characteristic of the individual, but also, and mainly, something that depends on family and community conditions and on the organizations that offer health and social services, making it a determinant of health.

Is it possible to measure HL in its different components? And how can we measure it at an individual, community or at an organizational level? These topics have been well described by Batterham and coll. in a review published in $2016^{[1]}$. The questions herewith expressed are not only a theme of research, because the assessment of $\mathrm{HL}$ level of individuals and populations can be used to improve the distribution - and the access to - of healthcare services and the participation of the community to the planning and the realization of healthcare interventions to promote 
health, and more health for all.

The measure of $\mathrm{HL}$ is in fact strictly linked to the opportunity to ensure the appropriate answer to healthcare needs for all the people and to improve equity in access to care. The HL measurement tools can explore two main different settings: the personal HL of each individual, and the organizational $\mathrm{HL}$ of the healthcare system and its structures (table 1). While in the first case the focus is on the patient (or the citizen) accessing the healthcare system to find the best answer for his/her needs, in the second circumstance the focus is on the citizen as part of a community: in this case, the assessment is on the structural and managerial aspects adopted to plan interventions for health promotion, disease prevention, and healthcare as developed by the organizations. It regards all the community with the aim to avoid inequalities in access to care.

These two main categories could be better described in different levels as in the following table: 


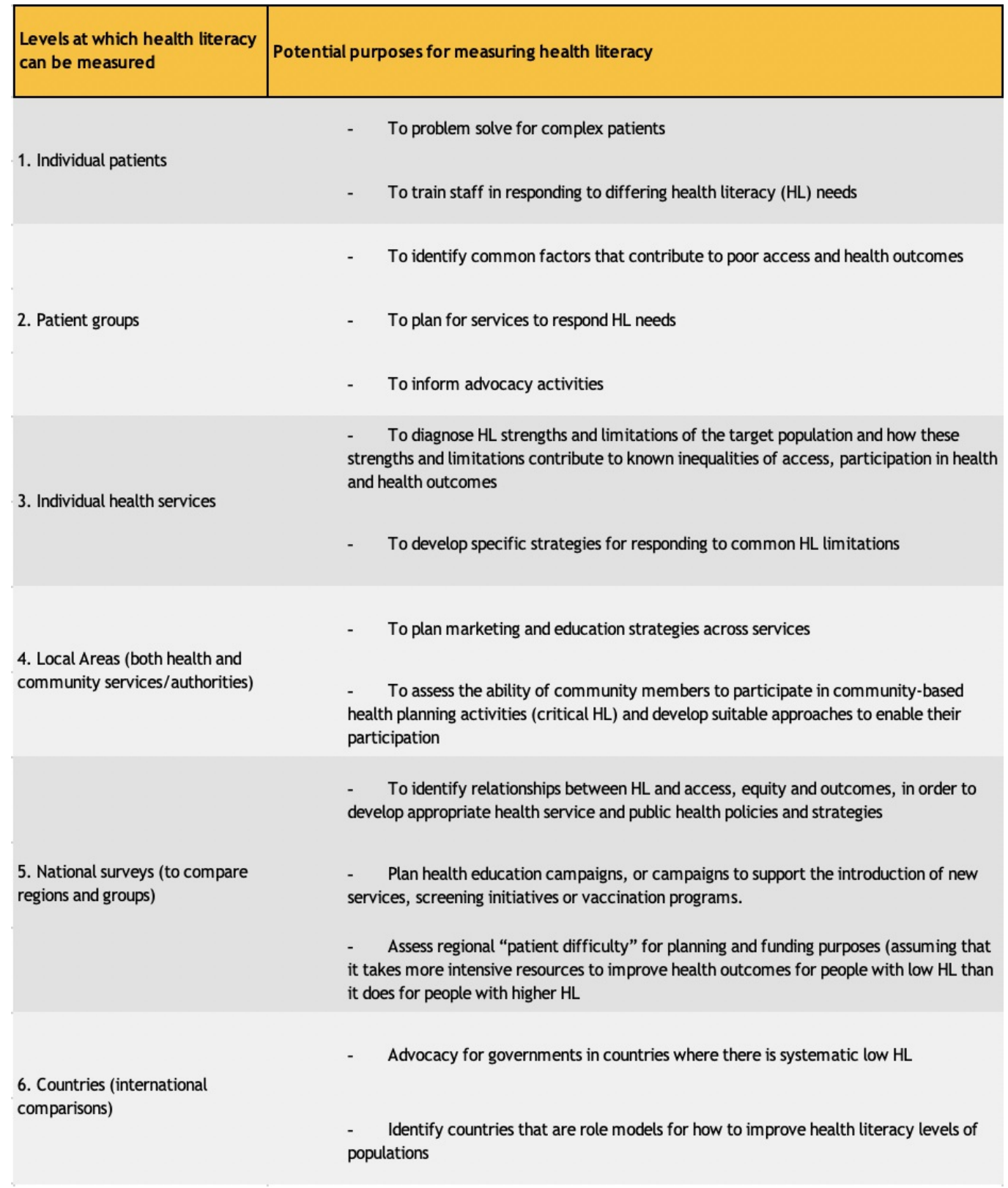

Table 1. Goals of the evaluations of health literacy levels of the health system and of the population. Adapted from ${ }^{[1]}$.

The assessment of the health literacy level can be done by means of specific instruments that have been developed in the last twenty-five years ${ }^{[2][3]}$ : they differ for the aim of the measurement (screening or deeper measurement), for the object (they use different definitions of health literacy and focus on one or more abilities), for the characteristics of the individuals (patients or population) and for the procedures used to evaluate their validity (i.e., face, content and concurrent validity compared to other instruments) ${ }^{[4]}$. The blooming of the productions of measurement tools is, on one hand an opportunity that gives more choices to researchers and operators for exploring specific issues, on the other hand it restricts the comparison between studies and populations because of 
the lack of a gold standard[5], which does not exist yet.

On a healthcare level, all the services must be appropriate and targeted to the HL level of the population. In order to do so, it is mandatory that the upper and middle management includes health literacy in the organizational policies and that all the healthcare workers are well informed about its role as a determinant of health. Specifically, if the people's or patients' HL level is not known, it is fundamental to apply some universal precautions to guarantee that all the persons will be able to understand the messages (prescriptions, services, professionals in charge, pathways) and will be well oriented in the healthcare environment ${ }^{[6]}$. This last feature is nowadays particularly important, considering the changes in the health systems on a national and regional levels and the growing complexity of services and places of healthcare, often far from the patients' residences and too hard to be comprised by themselves: see, for instance, the enormous increase in imaging and laboratory diagnostic services.

Among the universal precautions, interventions to improve written and oral communication have been carried on, like teach-back method or brown bag medication review techniques, and the use of easily understood written material, with few words and many images ${ }^{[6]}$.

At a managerial level (which corresponds to the third and fourth voices in table 1), a healthcare organization can be defined health literate if it is easily accessible for the population and if all the processes of distribution of services are patient-centered. Specifically, according to the Institute of Medicine ${ }^{[7]}$, a healthcare organization must respond to ten attributes to be defined as "health literate". A

Health Literate Health Care Organization

1. Has leadership that makes health literacy integ ral to its mission, structure, and operations.

2. Integrates health literacy into planning, evaluation measures, patient safety, and quality improvement.

3. Prepares the workforce to be health literate and monitors progress.

4. Includes populations served in the design, implementation, and evaluation of health information and services.

5. Meets the needs of populations with a range of health literacy skills while avoiding stigmatization.

6. Uses health literacy strategies in interpersonal communications and confirms understanding at all points of contact.

7. Provides easy access to health information and services and navig ation assistance.

8. Designs and distributes print, audiovisual, and social media content that is easy to 
understand and act on.

9. Addresses health literacy in high-risk situations, including care transitions.

10. Communicates clearly what health plans cover and what individuals will have to pay for services.

Furthermore, considering a bigger perspective on the health system, professionals must take into consideration that also the caregivers could have low health literacy levels; the studies done to evaluate this aspect highlight that half of the caregivers, both informal (that is, a familial, a parent or a friend) and formal (i.e., a paid person hired to take care of the patient) have inadequate health literacy levels ${ }^{[8]}$. Interventions to improve the health literacy level of caregivers must be encouraged, mainly for those patients with a low level of health literacy, for previous life experiences or intervening cognitive impairment. The health literacy level of those that are in contact with a person, either a caregiver or not - healthy, at risk or ill - can influence behaviours and health outcomes of both: the decision-making process is in fact influenced by all the people around us, and by the degree of knowledge the social network possess ad a whole, both because in touch with persons in need and in seek of health or because are leaders in the community in which the patient lives. (questo paragrafo, nonostante le mie molte correzioni/integrazioni, non mi convince).

At this proposal, Edwards and coll., in 2013, introduced the concept of distributed health literacy ${ }^{[9]}$, highlighting how HL could be distributed inside the social networks with the consequent possibility, for those with a low health literacy level, of using the knowledge of those around them to find, understand and use information about health. This process foresees that those who are more health literate contribute to help and improve the knowledge of those with a low health literacy level and to their acquisition of abilities, increasing in the end also their health literacy level. The relevance of distributed health literacy is culture-dependent and has to influence the strategies about how to improve the health literacy level in a community: the understanding of how debates are dealt with, how ideas are spread and how decisions are made should guide the choice of the target and to model the modes, time and setting of promoting, preventing and clinical pathways for those who need to be assisted in the healthcare and social contexts.

\section{The Austrialian experience}

In 2014 the Australian Commission on Safety and Quality in Health Care created a document aiming to sensitize the population and politicians on the importance of health literacy, in order to start a public debate on how to systematically tackle this topic, outlining stakeholders and their level of responsibility. The focus of this document is the necessity of developing this topic in an organized way, acting on health and non-health 
systems, working on communicational and educational (formal and informal) strategies. According to this prespective, OPtimising HEalth LIterAcy (Ophelia) is a community-based project started in Victoria that aims to identify and answer to health literacy needs of the population, acting both on an individual and on an organizational leve ${ }^{[10]}$. This approach, guided by eight principles (table 2), include three steps:

- assessment of the health literacy level of the population and of the healthcare structures;

- definition of interventions targeted to the needs, that have to be carried out on individuals, professionals, organizations or with the complete involvement of different organizations;

- improvement and evaluation of interventions.

The project has been adopted in nine services, comprising primary and homecare assistance as well as ICUs, creating the fit-for-purpose interventions, with the aim to improve organizational processes and staff knowledge and competencies, to involve the community and to obtain better health outcomes and equity among users.

\begin{tabular}{|l|l|}
\hline 1. Outcome focused & Improved health and reduced health inequities \\
\hline 2. Equity driven & $\begin{array}{l}\text { All activities at all stages prioritise disadvantaged group and those } \\
\text { experiencing inequity in access and outcome }\end{array}$ \\
\hline 3. Co-designed approach & $\begin{array}{l}\text { In all activities at all stages, relevant stakeholders engage collaboratively } \\
\text { to design solutions }\end{array}$ \\
\hline $\begin{array}{l}\text { 4. Needs-diagnostic } \\
\text { approach }\end{array}$ & Participatory assessment of local needs using local data \\
\hline 5. Driven by local wisdom & $\begin{array}{l}\text { Intervention development and implementation is grounded in local } \\
\text { experience and expertise }\end{array}$ \\
\hline 6. Sustainable & Optimal health literacy practice becomes normal practice and policy \\
\hline 7. Responsiveness & $\begin{array}{l}\text { Recognise that health literacy needs and the appropriate responses vary } \\
\text { across individuals, contexts, countries, cultures and time }\end{array}$ \\
\hline 8. Systematically applied & $\begin{array}{l}\text { A multilevel approach in which resources, interventions, research and policy } \\
\text { are organised to optimise health literacy }\end{array}$ \\
\hline &
\end{tabular}

Table 2. Principles of OPHELIA process (OPtimising HEalth LIteracy and Access), that guide the objectives, the Development and the implementation of interventions aiming to increase health literacy and equity inside communities. Adapted from ${ }^{[11]}$.

Final considerations

The field of health literacy is an open field to researches and studies; it is wide and still 
needs to be explored in its assumptions. The examples coming from the Anglo-Saxon Countries and the interventions made to assess and improve the health literacy levels demonstrate that the scientific world should consider this discipline as a key to improve the health of each single person and of communities, and they should push - but not only - healthcare organizations to shift to a real citizen-centered vision, never fully embraced until now in spite of the many promises pronounced both by politicians and healthcare professionals and managers.

References

1. a, b R.W. Batterham, M. Hawkins, P.A. Collins, R. Buchbinder, R.H. Osborne. (2016). Health literacy: applying current concepts to improve health services and reduce health inequalities. Public Health, vol. 132, 3-12.

2. ^ Sibel Vildan Altin, Isabelle Finke, Sibylle Kautz-Freimuth, Stephanie Stock. (2014). The evolution of health literacy assessment tools: a systematic review. BMC Public Health, vol. $14(1)$

3. ^ Jolie N. Haun, Melissa A. Valerio, Lauren A. McCormack, Kristine Sørensen, MichaelK. Paasche-Orlow. (2014). Health Literacy Measurement: An Inventory and Descriptive Summary of 51 Instruments. Journal of Health Communication, vol. 19 (sup2), 302333.

4. ^Joanne E. Jordan, Richard H. Osborne, Rachelle Buchbinder. (2011). Critical appraisal of health literacy indices revealed variable underlying constructs, narrow content and psychometric weaknesses. Journal of Clinical Epidemiology, vol. 64 (4), 366-379.

5. ^ Nguyen TH, Paasche-Orlow MK, McCormack LA. (2017). he State of the Science of Health Literacy Measurement. Stud Health Technol Inform, vol. 240:17-33.

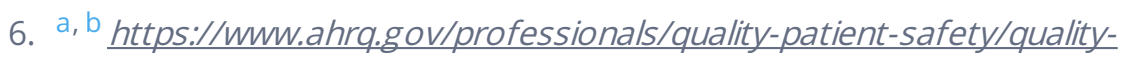
resources/tools/literacy-toolkit/index.htm/AHRQ Health Literacy Universal Precautions Toolkit. Content last reviewed August 2018. Agency for Healthcare Research and Quality, Rockville, MD

7. ^ Cindy Brach, Agency for Healthcare Research and Quality, Debra Keller, Lyla Hernandez, Cynthia Baur, Ruth Parker. (2012). Ten Attributes of Health Literate Health Care Organizations. NAM Perspectives, vol. 02 (6)

8. ^ Eva Y. N. Yuen, Tess Knight, Lina A. Ricciardelli, Susan Burney. (2016). Health literacy of caregivers of adult care recipients: A systematic scoping review. Health Soc Care Community, vol. 26 (2), e191-e206.

9. ^ Michelle Edwards, Fiona Wood, Myfanwy Davies, Adrian Edwards. (2013). 'Distributed health literacy': Iongitudinal qualitative analysis of the roles of health literacy mediators 
and social networks of people living with a long-term health condition. Health Expect, vol. 18(5), 1180-1193.

10. ^ Roy W Batterham, Rachelle Buchbinder, Alison Beauchamp, Sarity Dodson, Gerald R Elsworth, Richard H Osborne. (2014). The OPtimising HEalth LIterAcy (Ophelia) process: study protocol for using health literacy profiling and community engagement to create and implement health reform. BMC Public Health, vol. 14 (1)

11. ^Alison Beauchamp, Roy W. Batterham, Sarity Dodson, Brad Astbury, Gerald R. Elsworth, Crystal McPhee. (2017). Systematic development and implementation of interventions to OPtimise Health Literacy and Access (Ophelia). BMC Public Health, vol. $17(1)$ 